\title{
Impact of COVID-19 on the Clinical Picture and Therapy of Schizophrenia
}

\author{
Nadir A. Aliyev ${ }^{*}$, Teymur A. Qafarov ${ }^{1}$, Agahasan R. Rasulov', Eldar R. Hagverdi ${ }^{1}$ and Zafar N. Aliyev $^{2}$ \\ ${ }^{1}$ Department of Psychiatry and Drug Addiction, Azerbaijan State Advanced Training Institute, Baku, Azerbaijan. \\ ${ }^{2}$ Azerbaijan Medical University, department of psychiatry Baku, Azerbaijan.
}

*Corresponding Author: Nadir A. Aliyev, Department of Psychiatry and Drug Addiction, Azerbaijan State Advanced Training Institute, Baku, Azerbaijan.

Received date: January 10, 2022; Accepted date: January 28, 2022; Published date: February 08, 2022

Citation: Nadir A. Aliyev, Teymur A. Qafarov, Agahasan R. Rasulov, Eldar R. Hagverdi and Zafar N. Aliyev. (2022). Impact of COVID-19 on the Clinical Picture and Therapy of Schizophrenia. J Clinical Research and Reports, 10(3); DOI:10.31579/2690-1919/229

Copyright: (C) 2022, Nadir A. Aliyev. This is an open access article distributed under the Creative Commons Attribution License, which permits unrestricted use, distribution, and reproduction in any medium, provided the original work is properly cited.

\section{Abstract}

Objective: The purpose of this study was a retrospective study of the contingent of schizophrenia patients who were most infected with COVID-19.

Materials and Methods: We study 350 patients schizophrenia who infected with COVID-19 impact on the clinical picture and therapy of the illness. The investigation ware carried out in psychiatric hospital Ministry of Health of the Republic of Azerbaijan. The hospital has a capacity of 2000 beds.

Results: patients with schizophrenia who infected with COVID-19 in these clinically observed following changes: the intensity of psychopathological symptoms decreases, psychosomatic balancing occurs, that is, during the COVID19 illness, schizophrenic symptoms fade into the background, practically become invisible.

Conclusion: Patients schizophrenia who infected with COVID-19 in these clinically observed shows an improvement in their mental health.

Keywords: schizophrenia; infected with COVID-19; psychopathological symptoms; hospital setting

\section{Introduction}

Current statistics on coronavirus as of $01 / 08 / 2022$ (worldwide)

Total infections 303984489

Fatalities 5,499,350 (1.8\%)

Recovered 258408209 (85.0\%)

Now 40,076,930 are ill (13.2\%)

Current statistics on coronavirus as of 8.01.2022 (Azerbaijan)

Population 10,278 thous.

Total infections $6206796.0 \%$

Fatalities $8,4371.4 \%$

Recovered 605,058 97.4\%

Now 7184 are ill (1.2\%)
COVID-19 Pandemic causes numerous mental disorders. In our early studies, we showed the mental disorders caused by the COVID-19 Pandemic [1-5].

\section{Materials and methods}

We searched eight databases with search terms relating to COVID-19 and schizophrenia:

\section{MEDLINE}

\section{PreMEDLINE}

Cochrane Library

http://www.ema.europa.eu/ema/

https://scholar.google.com

https://www.rxlist.com/script/main/hp.asp

http://www.nejm.org 
https://www.bmj.com

Which would reflect the data of which mental disorder is most likely to have COVID-19 infection. However, we did not find any data impact of COVID-19 on the clinical picture and therapy of schizophrenia.

Retrospective study 350 (all men) clinical record in psychiatric hospital, the Ministry of Health of the Republic of Azerbaijan. The study was carried out from March 2020 to December 2021. The age of the patients ranged from 17 to 65 years. Most of the patients were 25-65 years old. The diagnosis was made using DSM-5.

The diagnosis was made using DSM-5. The mental state of the patients with was schizophrenia determined by the Positive and Negative Syndrome Scale (PANSS) before and after COVID-19 according to the history of the disease. Showed that the severity of psychopathological symptoms decreased 2-3 times. That is, as if the patients were in a state of remission, during the time of the disease COVID-19. After recovery from COVID-19, the previous symptoms of schizophrenia returned, but less intensely. In patients with schizophrenia, not a single death was noted.

\section{Discussion}

As is known from the literature, researchers mainly studied the prevalence of COVID-19 mainly in outpatients. At the same time, it was found that among mental patients, COVID-19 most often suffer from homeless people and people with mental retardation [6-10].

We consider it important to provide information on mental and somatic balance in order to clarify the psychopathological changes that have occurred in patients with schizophrenia with COVID-19.

Thus, it is clear that "pure" schizophrenia can manifest itself as a link between psychosomatic diseases (gastric duodenal ulcer, asthma, hypertension, etc.). But unlike somatic diseases, schizophrenia is reversible, i.e. the process is functional.

Finally, the somatic disorders caused by COVID-19 completely cover the clinical picture of schizophrenia, and the mental component is no longer understood by patients. This schizophrenia is extremely difficult to detect or undetectable. In essence, the milder the severity of schizophrenia, the more intense the somatic manifestations. On the contrary, schizophrenia replaces the expressive somatic component (imitation of somatic symptoms).

Thus, the somatic disorders caused by COVID-19 are observed in the psychosomatic balance of the clinical picture of schizophrenia. Krasnushkin EK [11]. Is considered a general law for mental and somatic diseases.

During this transition, an interesting event occurs - the disorder of true schizophrenia disappears in the patient's mind. This begs the question of whether it is appropriate to use small doses of medication in schizophrenia if somatic disorders come to the fore.

\section{Conclusion}

Thus, for the first time we carried out a retrospective analysis, it showed that patients schizophrenia who infected with COVID-19 in these clinically observed shows an improvement in their mental health. This begs the question of whether it is appropriate to use small doses of medication in schizophrenia if somatic disorders come to the fore.

The limitation of our work is as follows: 1) it is necessary to carry out such studies in many centers; 2) similar research needs to be carried out on the female contingent; 3 ) on a large clinical material.

\section{Conflict of Interest Statement}

The authors declare that the research was conducted in the absence of any commercial or financial relationships that could be construed as a potential conflict of interest

\section{Author Disclosure Information}

The authors declare that the article is submitted on behalf of all authors. None of the material in the article has been published previously in any form and none of the material is currently under consideration for publication elsewhere other than noted in the cover letter to the editor. Authors declare no financial and personal relationship with other people or organizations that could inappropriately influence this work. All authors contributed to and have approved the final article. The authors declare no conflicts of interest. No sponsor provided funding for this study.

\section{Acknowledgments}

The authors would like to thank the team of doctors of the psychiatric hospital \# 1 Ministry of Health of the Republic of Azerbaijan.

\section{References}

1. Aliyev NA and Aliyev ZN. (2020). Mental Disorders Caused by the COVID-19 Pandemic. A Mental Disorders Caused by the COVID-19 Pandemic. Ann Psychiatry Ment Health 8(2): 1151.

2. Aliyev NA and Aliyev ZN. (2021). Clinical Features of A Depressive Episode in People Who Had and Haven't Had Covid-19. Adv Neur Neur Sci. 4(2): 1-4.

3. Nadir A Aliyev, Zafar N Aliyev. (2021). Alternative Treatment Of Anxiety Disorders During Covid-19 Pandemic Which Snepco. Clinical Psychology and Mental Health Care, 2(4).

4. Nadir A Aliyev and Zafar N Aliyev. (2021). Treatment of AlcoholDependencein theContext of theCOVID-19 Pandemic. J Drug Addi Ther; Volume 2(1):2-4.

5. NA Aliyev, ZN Aliyev. (2021). "Valproate (depakine- chrono) in the acute treatment of outpatients with benzodiazepine addiction therapy during COVID-19 pandemic: Randomized, double-blind placebo-controlled study", Aditum Journal of Clinical and Biomedical Research, 1(1).

6. Bojdani E, Rajagopalan A, Chen A, Gearin P, Olcott W, Shankar V, et al. (2020). COVID-19 Pandemic: Impact on psychiatric care in the United States. Psychiatry Res. $2020 \mathrm{Jul}$; 289:113069.

7. Kar SK, Arafat SMY, Marthoenis M, Kabir R. (2020). Homeless mentally ill people and COVID-19 pandemic: The two-way sword for LMICs. Asian J Psychiatr. $2020 \mathrm{Apr}$ 10;51:102067.

8. Li S, Zhang Y. (2020). Mental healthcare for psychiatric inpatients during the COVID-19 epidemic. Gen Psych. 2020 Apr 1; 33(2):e100216.

9. Shinn AK, Viron M. (2020). Perspectives on the COVID-19 Pandemic and Individuals with Serious Mental Illness. J Clin Psychiatry. 2020 Apr 28; 81(3).

10. Yahya AS, Khawaja S, Chukwuma J. (2020). The Impact of COVID-19 in Psychiatry. Prim Care Companion CNS Disord. 2020 Apr 16; 22(2).

11. Krasnushkin E. K. (1960). About some relationships between mental and somatic diseases (selected diseases). M., 1960. P.427-445 (in Russian). 